\title{
Mindfulness, imagen corporal y prevención de los trastornos alimentarios en la \\ adolescencia
}

\author{
Rosa Marianela Salamea-Nieto, Juan Carlos Fernández-Méndez, Belén Prieto-Castaño y Ma Ángeles González- \\ Fernández \\ Facultad de Ciencias de la Educación, Universidade da Coruña
}

\begin{abstract}
Resumen
El presente análisis bibliográfico tiene como objetivo realizar una revisión de la literatura acerca de la utilidad del mindfulness en el fortalecimiento de la imagen corporal y su papel preventivo en los trastornos alimentarios entre adolescentes. Dicha revisión se llevó a cabo en la base de datos PsycINFO, localizándose un total de 88 trabajos publicados desde 1985 hasta la actualidad. Únicamente uno de los trabajos reunía todos los criterios de la búsqueda $\mathrm{y}$, por tanto, la principal conclusión que se extrae es la necesidad de realizar investigaciones en este campo.
\end{abstract}

Palabras clave: Mindfulness, imagen corporal, trastornos alimentarios, programas de prevención, adolescencia.

El éxito de la prevención de los trastornos alimentarios (TA) representa un objetivo importante debido a los efectos perjudiciales a largo plazo sobre la salud y el bienestar personal, así como los modestos resultados alcanzados con el tratamiento. Con esta finalidad, se lleva a cabo un análisis bibliográfico que permita examinar la literatura relacionada con programas preventivos en población adolescente y que se valen del mindfulness para facilitar la aceptación de la propia imagen corporal. Las actitudes hacia la imagen corporal juegan un papel nuclear en la aparición de TA (Merino, Pombo y Godás, 2001; Trujano, Nava, de Gracia, Limón, Alatriste y Merino, 2010), especialmente en la anorexia y la bulimia nerviosa que constituyen dos de las psicopatologías con mayor índice de mortalidad (Toro, 2004).

Los conceptos de bello y de feo están en relación con los distintos períodos históricos o las distintas culturas. En este sentido Eco (2004) señala en su obra que a lo largo de los siglos, filósofos $\mathrm{y}$ artistas han ido proporcionando definiciones de lo bello y, gracias a sus testimonios, ha sido posible la reconstrucción de una historia del ideal estético. Dicho ideal ha derivado a través de los tiempos hasta el concepto actual de belleza de consumo, en donde los medios de comunicación de masas juegan un papel fundamental en la exaltación del culto al cuerpo. Esa actitud genera en nuestra sociedad occidental una respuesta que lleva a la veneración de lo considerado bello, armónico y estético como contrapuesto de lo feo (Eco, 2007). Esa veneración por la belleza se traduce en una obsesión por un determinado ideal de la imagen corporal.

La imagen corporal supone un constructo multidimensional que representa la manera cómo se ven los individuos a sí mismos; los sentimientos e imágenes que les evoca dicha percepción (Raich, 2000). Un elemento central de la imagen corporal es la actitud hacia el propio cuerpo (Sepúlveda, Botella y León, 2001), definida como evaluación general (positiva o negativa) que las personas realizan de su cuerpo; cuando dicha valoración es negativa se hace más probable la aparición de un TA. Dichas actitudes afectan tanto a la conducta (por ejemplo, restricciones alimentarias), los pensamientos (una creencia, por ejemplo, de que un cuerpo delgado es un cuerpo atractivo) como a las emociones (principalmente, culpa o ansiedad) relacionadas con la comida (Gasco, Briñol y Horcajo, 2010).

Muchos adolescentes muestran una significativa insatisfacción con su propio cuerpo y, además, presentan sentimientos de culpabilidad por la comida que han ingerido aún cuando lo hayan hecho en cantidades saludables y razonables, tal y como pone de manifiesto una investigación desarrollada por Lameiras, Calado, Rodríguez y Fernández (2003). Por esa razón, es necesario el desarrollo de programas preventivos que permitan corregir estos aspectos en adolescentes y en jóvenes.

Los programas preventivos basados en métodos psicoeducacionales han permitido alcanzar mejoras en la imagen corporal (Jacobi, Beckers, Bronisch-Holtze, Winter y Winzelberg, 2007) así como en las puntuaciones en diferentes cuestionarios específicos sobre TA (O’brien y LeBow, 2007). En la misma línea, un metanálisis realizado por Cororve, Warren, CepedaBenito y Gleaves (2006) que incluía 46 estudios donde se clasificaban distintos tipos de programas preventivos (psicoeducativos y no psicoeducativos), permitió concluir que los diversos programas potenciaban, entre otros aspectos, la satisfacción corporal; además, no se apreciaron diferencias significativas entre los distintos programas. Sin embargo, otro metanálisis que incluía 68 estudios controlados sobre programas de prevención (Stice, Shaw y Marti, 2007), con objetivos bien distintos (entre ellos, la modificación de la distorsión del ideal de delgadez), mostró que un $51 \%$ de los mismos reducían los factores de riesgo de aparición de TA, y que los programas menos eficaces eran los que requerían una papel menos activo por parte del individuo. Por esa razón, algunos programas integran componentes que requieren un papel más activo por parte de los adolescentes. En la última década se ha empezado a 
considerar el papel activo que puede tener el mindfulness para ayudar a las personas a manejar alteraciones asociadas a problemas alimentarios (Bayón y Bonan, 2013).

El mindfulness se traduce al español como "atención plena" o "conciencia plena", y sirve para referirse (a) a un constructo teórico, (b) a una práctica para desarrollar atención plena, como es la meditación, o (c) a un proceso psicológico que implica estar consciente (Simón, 2015). Enfoques basados en el mindfulness, entendido como una forma de meditación, han recibido suficiente apoyo empírico como tratamiento para conseguir una mejoría en la sintomatología en los TA (véase, por ejemplo, Butryn, Juarascio, Shaw, Kerrigan, Clark, et al., 2013); así como en la construcción de una imagen corporal más positiva (Kristin, 2014), aunque escasean los trabajos que consideran la atención plena como una estrategia de prevención.

\section{Método}

El punto de partida de la búsqueda bibliográfica ha consistido en la localización de las publicaciones que abordan el tema a través de una selección informatizada en la base de datos PsycINFO. Dicha base de datos ha sido desarrollada por la American Psychological Association, y consta de más de 3.000 .000 de registros relacionados con la Psicología, Psiquiatría, Medicina y Trabajo social.

Las referencias se han localizado bajo los términos Eating disorders, mindfulness, body image, prevention programs y adolescence como palabras clave en los campos de búsqueda; para ambos géneros, en la adolescencia así como en todo tipo de publicaciones y para cualquier de fecha. Se descartaron todas las publicaciones que no estaban escritas en las lenguas inglesa o castellana.

\section{Resultados}

Primeramente se han computado los trabajos que se han obtenido con cada uno de los términos clave por separado. Las referencias que se han localizado bajo los términos mindfulness y meditación (mindfulness and meditation, M\&M), imagen corporal (body image, BI) y programas de prevención de los trastornos alimentarios (eating disorders prevention programs, EDPP) como palabras clave en los campos de búsqueda han sido numerosas, obteniéndose un total de 915 para M\&M, siendo la fecha de la publicación más antigua del año 1975; 17.553 para BI (con el año 1656 para la publicación más antigua), mientras que para el término EDPP se localizaron un total de 1.828, siendo el año 1957 la primera fecha de publicación de dicho tópico. En la Tabla 1 pueden apreciarse el número de revistas científicas, tesis doctorales y libros que se han escrito para cada uno los términos de la búsqueda llevada a cabo.

Dado el gran volumen de trabajos existentes y el interés del presente estudio bibliográfico únicamente se han tenido en cuenta los trabajos que centraban la investigación en los aspectos que relacionaban BI, EDPP y M\&M.

Una vez delimitados los campos de búsqueda, la evidencia que se desprende de la indagación bibliográfica es la escasez de estudios que relacionan todos los términos: mindfulness, imagen corporal y programas de prevención de los trastornos alimentarios. Se localizaron un total de 88 trabajos, que comprendían las fechas 19852015. En la Tabla 2 se aprecia una tendencia creciente en la comunidad científica por el interés por el mindfulness a partir del año 2008, siendo el 2012 cuando más trabajos se han publicado (17), si bien es cierto que todavía no ha concluido el 2015 y el número de trabajos podría ser superior al de esa fecha.

Tabla 1.

Resultados de la búsqueda en PsycINFO

\begin{tabular}{lccc}
\hline Palabra clave & $\begin{array}{c}\text { Revistas } \\
\text { científicas }\end{array}$ & $\begin{array}{c}\text { Tesis } \\
\text { doctorales }\end{array}$ & Libros \\
\hline $\begin{array}{l}\text { Body image } \\
\begin{array}{l}\text { Eating disorders prev. } \\
\text { prog. }\end{array}\end{array}$ & 13.058 & 2.431 & 2.064 \\
$\begin{array}{l}\text { Mindfulness and } \\
\text { meditation }\end{array}$ & 642 & 102 & 171 \\
\hline
\end{tabular}

Tabla 2.

Número de trabajos publicados desde 1985

\begin{tabular}{cc}
\hline Año de publicación & Número de trabajos \\
\hline 1985 & 1 \\
1986 & 1 \\
1997 & 1 \\
1999 & 1 \\
2000 & 1 \\
2004 & 2 \\
2005 & 1 \\
2007 & 1 \\
2008 & 6 \\
2009 & 7 \\
2010 & 10 \\
2011 & 9 \\
2012 & 17 \\
2013 & 12 \\
2014 & 13 \\
2015 & 5 \\
\hline
\end{tabular}

Se encontró únicamente un trabajo publicado en la revista científica International Journal of Eating Disorders (Atkinson y Wade, 2015) que reunía todos los criterios de la búsqueda: utilización del mindfulness como componente principal del programa, participantes menores de 18 años, centrado en la prevención y no en el tratamiento $\mathrm{y}$, por último, que la intervención incidiese en la imagen corporal, entre otros aspectos.

Dicho estudio tenía como objetivo evaluar la viabilidad y eficacia de un programa basado en la intervención de mindfulness con la finalidad de reducir el riesgo de aparición de los TA. Con esa finalidad, los autores del trabajo diseñaron un ensayo controlado aleatorizado que 
se llevó a cabo entre 347 chicas adolescentes $(\mathrm{M}=15.70$, $\mathrm{SD}=0.77$, que fueron asignadas a tres grupos: (a) grupo de intervención basado en el mindfulness $(\mathrm{N}=138)$, (b) grupo de intervención basado en la disonancia cognitiva $(\mathrm{N}=108)$ y (c) un grupo control $(\mathrm{N}=101)$. Los participantes tanto del grupo de intervención centrado en el mindfulness como los participantes del grupo basado en la disonancia recibieron el programa en tres sesiones. En la Tabla 3 pueden apreciarse los diferentes componentes del programa de mindfulness llevados a cabo en cada una de las sesiones.

Tabla 3.

Características del programa de mindfulness

\begin{tabular}{ll}
\hline \multicolumn{1}{c}{$\mathrm{N}^{\mathrm{o}}$ sesión } & \multicolumn{1}{c}{ Componentes } \\
\hline Sesión 1 & Estrategias de afrontamiento comunes: \\
(introducción al & ejercicios de supresión y ampliación \\
mindfulness) & Vídeo: "hoy es un regalo" (Kung Fu \\
& Panda) \\
& Presentación de diapositivas sobre lo \\
& que no es mindfulness \\
& Ejercicio guiado de conciencia presente \\
& Tareas para casa \\
& - Registro de estrategias de manejo \\
& Conciencia y aceptación de tareas \\
& rutinarias
\end{tabular}

Sesión 2 (nueva forma de relacionarse con las experiencias) compasión)
Sesión 3 (auto-

\author{
Impedimentos a la atención plena \\ Ejercicio de pensamiento centrado \\ Ejercicio dirigido: sentado con \\ imágenes de revista \\ Actitud de mindfulness en grupo \\ Método STOP para contrarrestar el \\ piloto automático \\ Tareas para casa \\ - Practicar STOP \\ - Conciencia de las experiencias \\ agradables del cuerpo
}

Analizar en parejas el poema "Guest House"

Torbellino de ideas de declaraciones de aceptación compasiva

Ejercicio guiado: visualizado del espejo de reflexión

Discussion de las presiones futuras, y desarrollar un plan de acción personal Tareas para cas

- Practicar STOP

Las participantes del grupo que recibieron las sesiones de mindfulness $(\mathrm{N}=156)$ mostraron reducciones significativas en sus preocupaciones por la percepción del peso y la figura corporal al ser comparadas con las participantes del grupo control. Finalmente, es importante señalar que no se encontraron diferencias al realizar comparaciones con el grupo sometido a la intervención basada en la disonancia cognitiva. Los autores del estudio concluyen que los resultados son prometedores en cuanto a la utilidad del mindfulness en la prevención de los TA; sin embargo, señalan que son necesarios más trabajos para analizar su eficacia frente a otros métodos preventivos.

\section{Discusión y conclusiones}

Los resultados de este análisis bibliográfico ponen de manifiesto la ausencia de investigaciones que consideran el mindfulness dentro de los componentes de los programas preventivos de $\mathrm{TA}$ en la población adolescente.

Siguiendo a Stice, Chase, Stormer y Appel (2001) es importante en el diseño de los programas preventivos de los TA dirigidos a población en riesgo, con el propósito de lograr la máxima efectividad; la utilización de métodos alternativos a los psicoeducativos tradicionales, utilizando otras técnicas que logren un cambio actitudinal, y basados en algún modelo etiológico validado empíricamente.

De un total de 88 trabajos publicados entre el 1985 y el 2015 sólo uno reunía todos los criterios de la búsqueda realizada (Atkinson y Wade, 2015). Por esa razón, sería necesario diseñar investigaciones realizadas en nuestro país de corte experimental que permitan comparar la eficacia del mindfulness orientado hacia la autocompasión (Neff, 2012) y que favorezcan la potenciación de una imagen corporal positiva para prevenir los TA.

\section{Referencias}

Atkinson, M. y Wade, T.D. (2015). Mindfulness-Based Prevention for Eating Disorders: A School-Based Cluster Randomized Controlled Study. International Journal of Eating Disorders. Published online in Wiley Online Library.

http://dx.doi.org DOI: 10.1002/eat.22416.

Bayón, C. y Bonan, V. (2013). Trastornos de la conducta alimentaria e intervenciones psicoterapéuticas que incorporan mindfulness (pp. 209-240). En V. Simón y M.T. Miró (Eds.): Mindfulness en la práctica clínica ( $2^{\text {a }}$ Ed.). Bilbao: Editorial Desclée de Brouwer.

Butryn, M.L., Juarascio, A., Shaw, J., Kerrigan, S.G., Clark, V et al. (2013). Mindfulness and its relationship with eating disorders symptomatology in women receiving residential treatment. Eating Behaviors, 14, 13-16.

Cororve, F.M.,Warren, C.S., Cepeda-Benito, A. y Gleaves, D.H. (2006). Eating disorder prevention research: a meta-analysis. Eating Disorders: The Journal Treatment \& Prevention, 14, 191-213.

Eco, U. (2004). Historia de la belleza. Buenos Aires: Ed. Lumen.

Eco, U. (2007). Historia de la fealdad. Buenos Aires: Ed. Lumen.

Gasco, M., Briñol, P., y Horcajo, J. (2010). Cambio de actitudes hacia la imagen corporal: el efecto de la elaboración sobre la fuerza de las actitudes. Psicothema, 22, 71-76.

Jacobi, C., Morris, L., Beckers, C., Bronisch-Holtze, J.,Winter, J. y Winzelberg A.J. (2007). Maintenance of internet-based prevention: a randomized controlled trial. International Journal of Eating Disorders, 40,114-119.

Kristin, E. (2014). Mindfulness and its relationship to body satisfaction and risk of an eating disorder in college women. Gredos. ProQuest Information \& Learning, AAI3599180. 
Lameiras, M., Calado, M., Rodríguez, Y. y Fernández, M. (2003). Hábitos alimentarios e imagen corporal en estudiantes universitarios sin trastornos alimentarios. Revista Internacional de Psicología Clínica y de la Salud, 3, 23-33.

Merino, H., Pombo, M.G., y Godás, A. (2001). Evaluación de las actitudes alimentarias y la satisfacción corporal en una muestra de adolescentes. Psicothema, 13, 539- 545.

Neff, K. (2012). Sé amable contigo mismo: El arte de la compasión hacia uno mismo. Barcelona: Ediciones Oniro.

O'Brien, K.M. y LeBow. M.D. (2007). Reducing maladaptive weight management practices: developing a psychoeducational intervention program. Eating Behaviors, 8, 195-210.

Raich, R.M. (2000). Imagen corporal. Madrid. Pirámide. Sepúlveda, A. R., Botella, J. y León, J. A. (2001). La alteración de la imagen corporal en los trastornos de la alimentación: un meta-análisis. Psicothema, 13, 7- 16.
Simón, V. (2015). Aprender a practicar mindfulness (11 Ed.). Barcelona: Sello Editorial.

Stice, E., Chase, A., Stormer, S., y Appel, A. (2001). A randomized trial of a dissonance-based eatingdisorders prevention program. International Journal of Eating Disorders, 29, 247-262.

Stice, E., Shaw, H. y Marti, C.N. (2007). A meta-analytic review of eating disorder prevention programs: encouraging findings. Annual Review of Clinical Psychology, 3, 207-231.

Toro, J. (2004). Riesgo y causa de la anorexia nerviosa. Barcelona: Editorial Ariel S.A.

Trujano, P. Nava, C., de Gracia, M., Limón, G., Alatriste, A.L. y Merino, M.T. (2010). Trastorno de la imagen corporal: Un estudio con preadolescentes y reflexiones desde la perspectiva de género. Anales de Psicología, 26, 279-287. 